\title{
Industrial Process Diagnosis based on Information-based
} Features

This paper was downloaded from TechRxiv (https://www.techrxiv.org).

\section{LICENSE}

CC BY 4.0

SUBMISSION DATE / POSTED DATE

18-02-2022 / 23-02-2022

\section{CITATION}

Aiordachioaie, Dorel (2022): Industrial Process Diagnosis based on Information-based Features. TechRxiv. Preprint. https://doi.org/10.36227/techrxiv.19195808.v1

DOI

10.36227/techrxiv.19195808.v1 


\title{
Industrial Process Diagnosis based on Information-based Features
}

\author{
Dorel Aiordachioaie \\ Dunarea de Jos University of Galati \\ 47 Domneasca \\ Galati, Romania \\ Dorel.Aiordachioaie@ieee.org
}

\begin{abstract}
Change detection and diagnosis based on information-based features is considered. As benchmark case study, the faults of bearings are selected. Information from the working states, including those generated by faults in bearings, are transformed and carried by the vibration's signals, which are further processed by advanced techniques of information and signal processing, as e.g., statistical models, Renyi and Tsallis entropies, and other complexity measures. These measures are considered features in the framework of pattern recognition and classification. The data records are explored with sliding windows, change detection being assigned at the window level. Diagnosis is solved via classification results based on similarity between the prototypes of the fault classes and the new data, which need classified. The method is tested with virtual and real recorded data from bearings with various type of faults. The results obtained on computer-based simulations show the feasibility of the considered method, and the limits of some information measures. The proposed method is general and is an alternative to other well known diagnosis methods based on process or signal models.
\end{abstract}

Keywords -- fault detection, process diagnosis, information, entropy, model, feature selection.

\section{INTRODUCTION}

This work is an extension of a conference paper, [1], which has been used an approach based on information and time domain model processing, all extracted from the vibration signals generated by faults in the elements of the rolling bearings. The approach is general and can be applied also to other various signals, associated or not with electrical and non-electrical (e.g., mechanical vibration) processes, and to other elements of industrial machineries and processes.

State change, fault detection and process diagnosis are important activities in industrial and process engineering, for many important tasks as condition monitoring, safety, or predictive maintenance.

In the field of fault detection and process diagnosis, two approaches are promoted, based on parity equations and, respectively, on signal processing paradigm, as described, e.g., in [2]-[8]. Both paradigms use models based on equations. The first one is a process model and the second one is a model of signal, directly from the analyzed process or residuals from other processing structures.

Depending of the working hypothesis when data is processed, the signal model-based for change detection and diagnosis (CDD) problems could consider various approaches to extract features as: (i) correlation coefficients and parameters of stochastic models (e.g. ARMA), for time domain; (ii) spectral analysis with or without models, for frequency domain; (iii) various transforms, as Short-TimeFourier-Transform (STFT), Wiener-Ville transform (WVT) or wavelet transform (WT), for time-frequency domain [9], [10]. Examples and details are available, e.g., in [11]-[14].

The basic relation among these approaches is presented in Fig.1, on two levels. At the bottom are methods based of signal-based models. These approaches can be solved with methods coming from three wide domains: time, frequency, and time-frequency domains, without excluding combinations among them. At the upper level, the information-based models are presented, as a new level of description, based on information representations and measures, e.g., entropies, starting from Shannon and continuing with Renyi, of various orders.

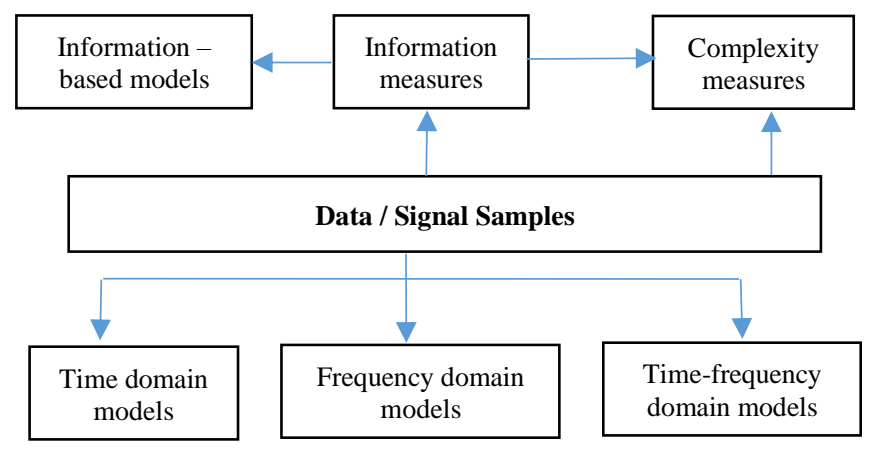

Fig. 1. The basic structure used in signal model- based CDD

Complexity can be used also in the description of the evolution of the signal / system under study. This can be described directly - in an independent way - based on specific elements of knowledge but can be also described in terms of probabilities distributions (in discrete case or probability density functions (in the continuous case), which are provided by information models of the source of the signals. An introduction and more explained examples and case studies are available in [14], [15], and [16].

Fault detection and diagnosis include many special methods as those based on statistical signal processing techniques, [17], variational autoencoder, [18], Bayesian networks, [19], or artificial intelligence, [20].

An important aspect is the sensitivity of the models to changes, as effects of the changes in the working conditions of the industrial process or as effect of the faults. The absolute value of the various criteria for CDD problems is not very important compared with the relative values, i.e., the difference among the values describing stationary regimes. This is the reason to search change detection criteria, which are sensitive to parameters used in the signal models, e.g., based on the variances of the variables/parameters used in models.

The present work use information-based models, i.e., models based on entropies and complexity of data processed. 
The entropies are considered as average measures of information computed / extracted from the processed data. For entropies, there are many information measures, e.g., Renyi entropy, and for complexity, the Lempel-Ziv complexity are just few examples. The information-based models are considered superior to classical methods based on signal processing and more general compared with other physical models, but - some time - the effective implementation and results could not be at the level of expectation. Very often, the model of the source or the hypothesis of data processing are not met, as is the case in many industrial environments. The information - based domain is exemplified with some examples as diversity entropy, [21], Kullbak-Leibler divergence, [22], dispersion entropy, [23], information fusion, [24], and probabilistic frameworks [25], [26].

The paper is organized in 6 Sections. The next section presents the basic solutions and signals involved in industrial process diagnosis, where vibrations are the most important effects of the faults. Section 3 describes the method, at the level of processing blocks and some details related to the implemented functions and algorithms for change detection and diagnosis (CDD). The information measures are presented as well. Two types of data are considered and presented in the section 4 . The first type corresponds to real measured signal. The second type is synthetized and based on the properties of the former. The results of the experiments based on computer simulation are presented in Section 5. Finally, the conclusion section presents the main results in a qualitative way and suggest some steps to follow.

\section{BASIC APPROACHES FOR VIBRATION SIGNALS}

Vibration and acoustic emissions are common effects of the incipient and advanced faults in machinery and intensively used as sources of signals. Focusing on rolling element bearings are discussed in [27] and [28].

The classic background of vibration - based monitoring is well presented in [29] and [30]. The state of the art related to the techniques used in fault diagnosis of the rolling element bearings is presented in [31], for signal processing techniques, and in [32], for data-driven approach. The interest for such elements (bearings) is explained by the widespread in electromechanical machineries and due to their importance in the safety of the processes and equipment, especially when high speed regimes and variable loads are involved. Some examples of processes with bearings are [33] - [37].

The effects of the (independent) faults in rolling element bearings could be estimated with relatively simple computation formula, based on information from the geometry and working conditions. However, in real industrial working conditions, with high amplitude and wide frequency range of the perturbations, the effects need more attention and advanced computation methods.

There is a variety of methods to detect faults in bearings, with methods in time, frequency, or time-frequency approaches, looking to a trade-off between performance and cost of the implementation. A wide set of advanced methods are available, as those based on machine learning [38]-[40], time-frequency framework [41], and synchrosqueezing transform [42].

Techniques based on information processing are used also to extract specific features from the analyzed signals. Basically, these are based on statistical and information models of data, which can use Renyi entropy measures of various orders and scales, and complexity measures as Lempel-Ziv, [43].

\section{DESCRIPTION OF THE METHOD}

The basic processing blocks in the information - based paradigm is presented in Fig. 2. The first block is for data preprocessing, which means filtering and scaling. Data are recorded in a buffer and explored with a non-overlapping sliding window of suitable length, $N w$, to sustain the trade-off between the performance of the point change detection and the stationarity of the analyzed signal, including data dynamic behavior (and transients). Working with non-overlapping windows could generate higher errors in the estimation process of the change point. This approach is less important if the sampling frequency is high and for the low frequency of the vibration signals, as in the case of the bearings of the ordinary rotating machines. Partial overlapping windows could be considered for an improvement of the detection accuracy, if necessary.

Data form the observation window is the input for the identification block, i.e., the block which generates the statistical model of the source associated with the analyzed data. The simplest discrete model is of zero-order and contains the symbols and the probabilities, in a pair of two vectors as $[\mathbf{S}, \mathbf{P}]$. The size of the model is Ns. The bigger the size, the more exact the model. The symbols are the (small, elementary) intervals of the dynamic range of the signal. The estimation of the probabilities could be developed by the histogram of data. More details are available in [44].

Once the model of the source is ready, the features can be considered and computed. The set of the features depends on the used paradigm, and can include features based on information measures, from time or frequency domains or a combination of these. The number of the features is $\mathrm{Nf}$, and depends on the values of other parameters, as Ns (the size of the model) and $N w$ (the size of the data window).

The feature selection stage decides which features are the most important for the task being implemented. There should be a criterion to select the best features. For example, a selection criterion based on the variance of the features could be a good starting candidate. The selection procedure depends also on the classifier results/performance. A simple classifier, e.g., one based on distance, provides good results only when a linear separation of the features is possible, which is not always the case.

The optimization block is on the feedback processing path. Based on the results provided by classifier, it should manage the parameters of the processing data chain, as $N f, N s$ and $N w$, in this order. Starting with some heuristic rules for the optimization process, including simple software-based tools, the computation of these parameters must be automated and/or under machine learning paradigm.

\section{A. Source model}

A zero-order model is considered for the source of the generated data, with parameters the symbols and the associated probabilities. Let be the dynamic range of data in the real valued interval, $I=[-A, A]$ with $A \in \mathcal{R}^{+}$. A uniform partition/grid with $n$ subintervals $i_{j}$ of length $q$ is considered as

$$
Q=\left\{i_{1}, i_{2}, \ldots, i_{j}, \ldots, i_{n}\right\}
$$




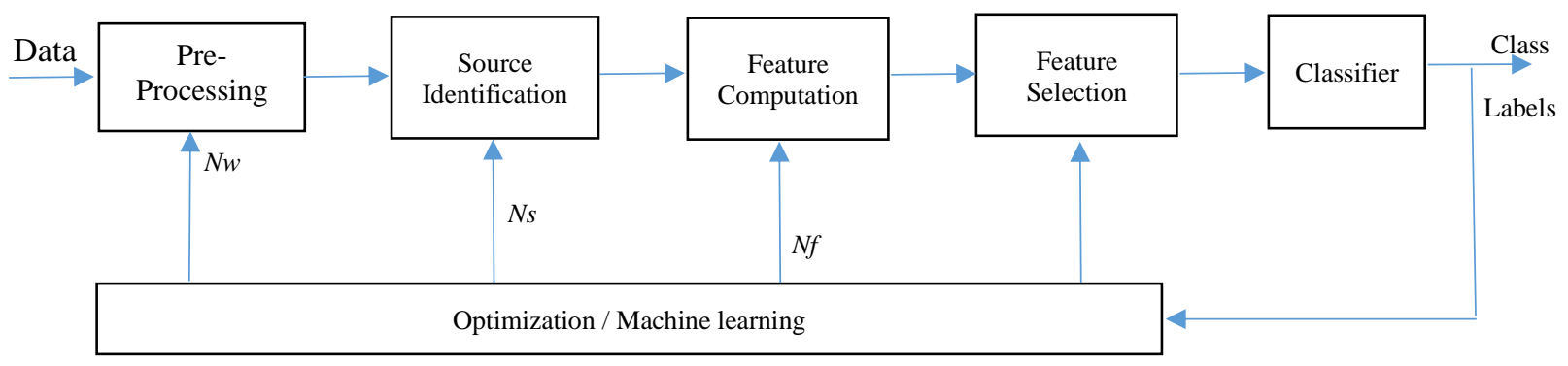

Fig. 2. CDD based on information processing paradigm

with

$$
i_{j} \cap i_{k}=\emptyset, \forall j, k=\overline{1, n}
$$

and

$$
\bigcup_{j=1}^{n} i_{j}=I
$$

The rule used in defining the symbol associated to an interval is

$$
\text { if } x_{k} \in i_{j} \rightarrow\left(x_{k}, s_{j}\right)
$$

which means the value $x_{k}$ is associated with symbol $s_{j}$. Thus, the elements of the alphabet of the source $S=$ $\left[\begin{array}{llllll}s_{1} & s_{2} & \ldots & s_{j} & \ldots & s_{n}\end{array}\right]$ is computed. By considering the histogram with a partition $Q$ over the realization(s) of time data series, the set values of the probabilities and later of the probability density function can be considered for analysis and classification purposes.

\section{B. Feature description}

The main information processing block computes a set of variables/features as estimations of Shannon entropy, Renyi entropy on various scales, Tsallis entropy, and Lempel - Ziv complexity, which are listed and briefly described in Table I. Depending on problem (e.g. the observation noise at the level of measurements), other general features could be included in the vector of the features, as the variance of the probabilities, the crest factor, the peak values, but these are not considered here.

The source model (which generated data) is a statistical model composed from the probabilities of the symbols. In the case of the signals, the symbols are the elementary intervals from the dynamic range of the signal (obviously, $[-1,1]$ ). The set of the probabilities could be used directly as set of features, if necessary.

The Renyi entropy of order $\alpha$, (HR), [45], is intensively used in many applications based on its basic properties as entropy, and on the generalization of the Shannon approach. Obviously, the orders 2 or 3 are used, but high orders are used when high variance of the output is necessary.

The multiscale entropy (MSRE) uses the estimation of the entropy over a range of scales [46], [47], and uses an averaging process of the values obtained on different timescales. Tsallis entropy, [48] [49], is another measure of the information content of a system or signal.
Complexity is an important feature in data analysis and structure description, which is evaluated here by using Lempel-Ziv complexity measure, [50].

The basic vector of the features, $\boldsymbol{F}$, contains five features from the information domain, $\boldsymbol{F I}$ (four entropies plus the Lempel-Ziv complexity) and three features, $\boldsymbol{F T}$, the variance, the skewness, and the kurtosis, applied to the set of the probabilities $\boldsymbol{P}$ :

$$
\boldsymbol{F}=[\boldsymbol{F I}, \boldsymbol{F T}]=[H S, H R, M S R E, H T, L Z V, V, S K, K]
$$

\begin{tabular}{|c|c|c|}
\hline No. & $\begin{array}{c}\text { Name of the } \\
\text { feature }\end{array}$ & Variable / expression \\
\hline 0. & Source model & $S:\left(\begin{array}{ccc}s_{1} & \ldots & S_{N} \\
P\left(s_{1}\right) & \ldots & P\left(s_{N}\right)\end{array}\right), \quad \sum_{i=1}^{N} P_{i}=1$ \\
\hline 1. & $\begin{array}{c}\text { Shannon } \\
\text { Entropy (SE) }\end{array}$ & $H(\boldsymbol{P})=-\sum_{i=1}^{N} P_{i} \cdot \log \left(P_{i}\right)$ \\
\hline 2. & $\begin{array}{l}\text { Renyi Entropy } \\
\text { or order } \alpha \text { (RE) }\end{array}$ & $H_{\alpha}(\boldsymbol{P})=\frac{1}{1-\alpha} \log \sum_{k=1}^{N} P_{k}^{\alpha}, \alpha>0$ \\
\hline 3. & $\begin{array}{l}\text { Multiscale } \\
\text { Renyi entropy } \\
\text { (MSRE) }\end{array}$ & $\begin{array}{c}y(j)^{(\tau)}=\frac{1}{\tau} \sum_{i=(j-1) \tau+1}^{j \tau} x(i), \quad 1 \leq j \leq \frac{N}{\tau} \\
\operatorname{MSRE}(\text { data })=H(Y)\end{array}$ \\
\hline 4. & Tsallis entropy & $H_{\alpha}(\boldsymbol{P})=\frac{1}{1-\alpha}\left(\sum_{k=1}^{N} P_{k}^{\alpha}-1\right), \alpha>0$ \\
\hline 5. & $\begin{array}{l}\text { Lempel Ziv } \\
\text { complexity (LZC) }\end{array}$ & - \\
\hline
\end{tabular}

TABLE I. INFORMATION BASED FEATURES

Once detection is made, a classification process of faults can be considered to implement the process diagnosis. A standard classifier based on similarities is considered, i.e., based on a distance measure. If $\boldsymbol{P}_{\boldsymbol{j}}$ is the prototype vector of the class $j$, and $\boldsymbol{b}$ is the tested feature vector, the classifier selects the class $k$ which is closest to $\boldsymbol{b}$, by using the equations below, i.e., it takes the minimum distance between $\boldsymbol{b}$ and $\boldsymbol{P}_{j}$

$$
k=\underset{j}{\operatorname{argmin}}\left\{d\left(\boldsymbol{b}-\boldsymbol{P}_{j}\right), j=0,1,2,3\right\}
$$

The results obtained with above features could be compared with techniques based on statistical correlation coefficients, [51], or Renyi divergence, [52]. For two vectors $\boldsymbol{a}$ and $\boldsymbol{b}$ of the same size, with elements samples of the signals or samples of the probability distributions, the above measures are defined as 


$$
R[k]=E[a[i] \cdot b[i+k]]=\frac{1}{N} \sum_{i=1}^{N} a[i] b[i+k]
$$

The Renyi divergence (RD) is estimated by:

$$
R D_{\propto}\left(\boldsymbol{P}_{i}, \boldsymbol{P}_{j}\right)=\frac{1}{\propto-1} \log \sum_{j=1}^{N} P_{I}(i)^{\propto} P_{J}(j)^{1-\propto}
$$

\section{DATA DESCRIPTION AND ANALYSIS}

\section{A. Data}

The process under study is an electric machine, with support elements based on bearings. Data were considered for the case of faults in the elements of the rolling bearings, available from [53], which are also explained and analyzed in [54]. Three types of faults are considered: inner race (F1), ball (F2) and outer race (F3). The case F0 means no faults. Each fault is associated with a pattern class. Finally, a case study with four classes is considered, from $\mathrm{C} 0$ to $\mathrm{C} 3$. The sampling rate is $12 \mathrm{kHz}$, and the electric motor is free of load.

The raw data matrix $\boldsymbol{D}$ for testing is defined with 4 vectors, randomly selected from each class, from \#0 to \#3. The size of $\boldsymbol{D}$ is $N \times(4+4+4+4)=N \times 16$. This will generate a matrix of prototypes, $\boldsymbol{P}$, of size $N \times 4$, one column for a class. The prototype vector is the average of the available signal vectors from a given class. Each column of matrix $\boldsymbol{D}$ defines a test case.

\section{B. Virtual Data}

Based on the behavior of the measured data, virtual data is defined and used in testing, by using additive white Gaussian noise $\boldsymbol{n} \sim N(0,1)$ to harmonic components. The frequencies of these components are in the frequency bandwidth where the real signals are. Such an approach is useful to learn/asses the difference between real and virtual data and to see the effects the CDD results. The signals are computed by

$$
s_{i}(t)=A \cdot \sin \left(2 \pi f_{i} \cdot t\right)+n(t), \quad i=0,1,2,3
$$

with $A=1$, and $\boldsymbol{f}=[1.0 ; 3.5 ; 3.0 ; 2.5][\mathrm{kHz}]$. The same data format as the previous (real) case is used, i.e., the data matrix $\boldsymbol{D}$ have the same dimension $N \times 16$, but the vectors of each class differ on signal-to-noise ratio (SNR). Fig. 3 presents an example for the power spectral densities of the prototype vectors, with $0.5 \mathrm{~dB}$ Signal-to-Noise-Ratio (SNR).

\section{Real Data Analysis}

Data analysis is important to consider assessing the complexity of the CDD problem, by proposing the number of the processing steps, and which are the parameters in each stage. In the case of real data, the analysis in time domain allows us to estimate the dynamic range of the signals and to estimate the power of the observation noise, under various loads and working conditions. The real physical signals show complex waveforms (impulsive components), which will require complex features to describe the shape of the signals for a right classification.
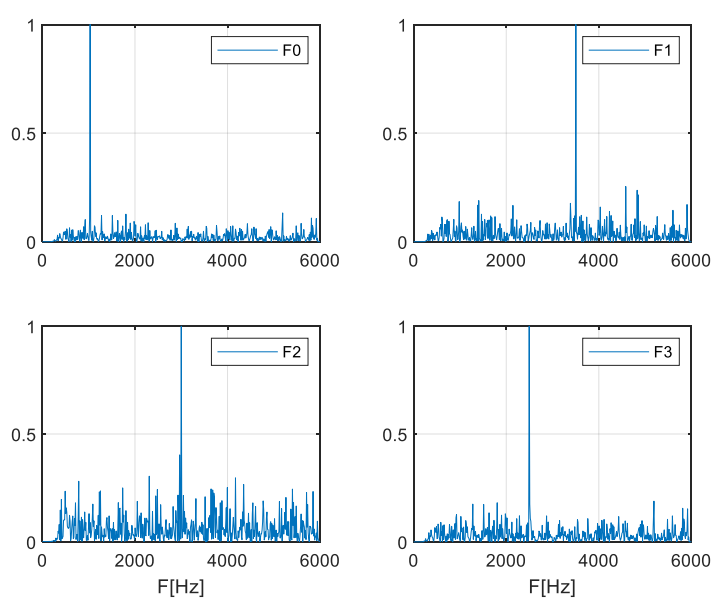

Fig. 3. Virtual data for testing. Example.

Fig. 4 presents the power spectral density for the prototype vectors of the four classes/faults (F0, F1, F2, and F3). A short visual analysis could reveal major superpositions among the power spectra of the vibration signals coming from different faults, and thus some difficulties in using methods in the frequency domain for CDD.
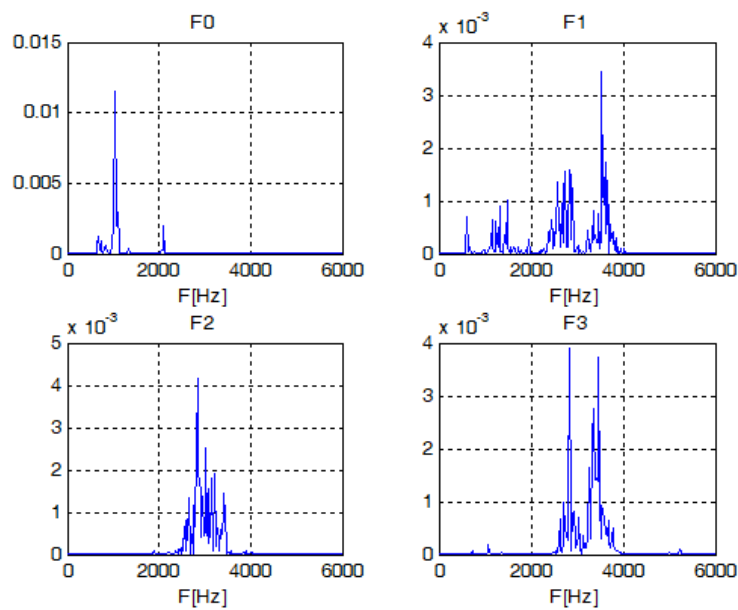

Fig. 4. Prototype vectors in the frequency domain for real data

The CDD method has some parameters (as $N s$ - the model order, $N w$ - the length of the observation window, $N f$ - the number of the features), which need optimizing for best performance. High rates of correct classification need high values of the size of the source model), and the point change detection accuracy requires small lengths of data window. The above parameters are not independent, i.e., a good model needs high values of $N s$ but also a greater window size, $N w$, to make a good identification and description of the signal.

\section{RESULTS OF THE EXPERIMENTS}

In the pre-processing stage, the physical data are passband filtered with a Butterworth type filter of order 5 and a frequency bandwidth between 100 and 6,000 Hz. A test vector composed of four segments; each o length 10,000 samples is considered. Data are scaled to the range of $[-1,1]$. Fig. 5 presents the probability distributions for data windows with indexes $i=1,11,22,31$ (from a set of $1 \ldots 40$ ), which correspond to different faults. The distributions have different shapes/parameters and could be used as descriptors/models of the faults. 

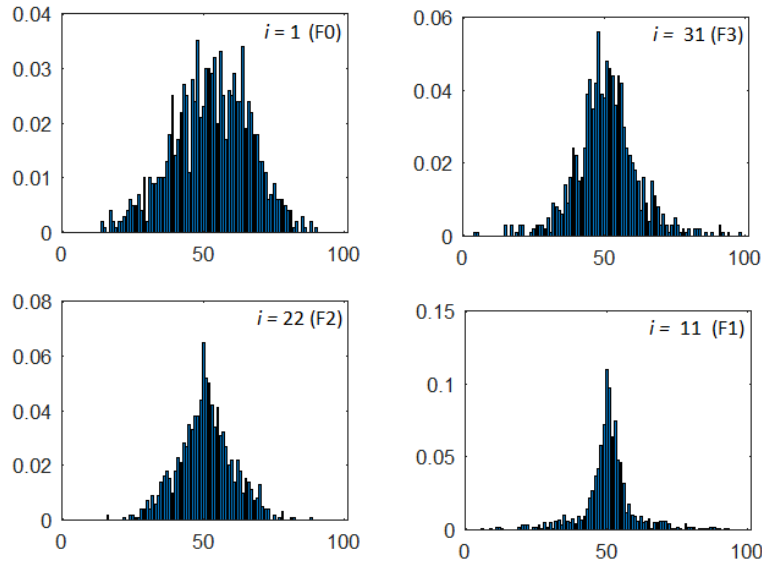

Fig. 5. Distribution of the probabilities for various faults

For a window length of 1,000 samples, $N w=40$ windows are obtained. Fig. 6 presents the evolution of the normalized features $\boldsymbol{F}$ for the real data test vector. The features do not have the same sensitivity to data changes, so a selection procedure is necessary. In this work, the feature selection is based on the variance of the features, as presented in Fig. 6, at the bottom side. Thus, the features with lowest variance could be removed from the feature vector going to classifier. The order of the features is:

$$
\boldsymbol{F}=[V, S K, K, L Z V, M S R E, H R, H S, H T]
$$
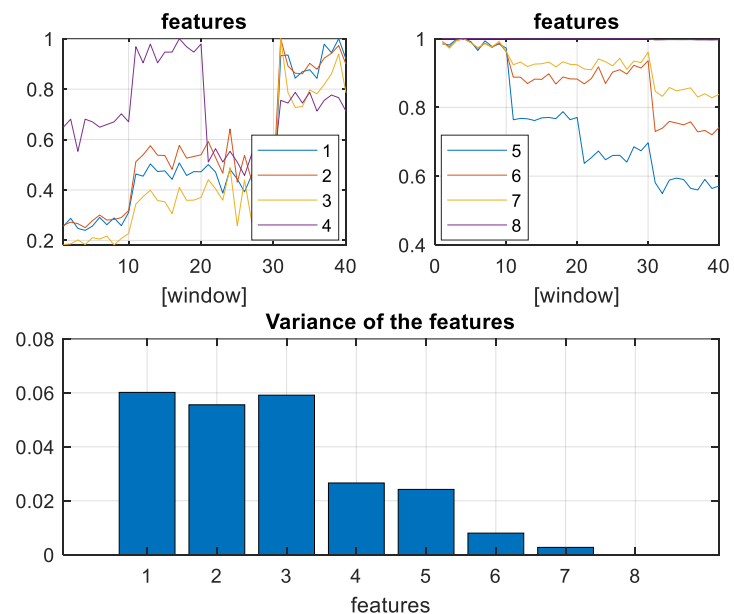

Fig. 6. Evolution of the features $\boldsymbol{F}$ (physical data)

Fig. 7 shows an example from the case of synthetic signal tests. On the left side, the evolution of the features is presented, for ordered features vector (Eq. 10). The relative different values indicate the dissimilarity of classes and the virtual success of the diagnosis, even for low size of the source model, i.e., $N s$ starting from 10 . On the right side, the output of the classification stage is presented, for eight observation windows. The classification is correct, i.e., the correct recognition rate is $100 \%$. A model size of $N s=100$ and the window's length $N w=5,000$ samples were used. A high value of SNR was used, about $18 \mathrm{~dB}$.
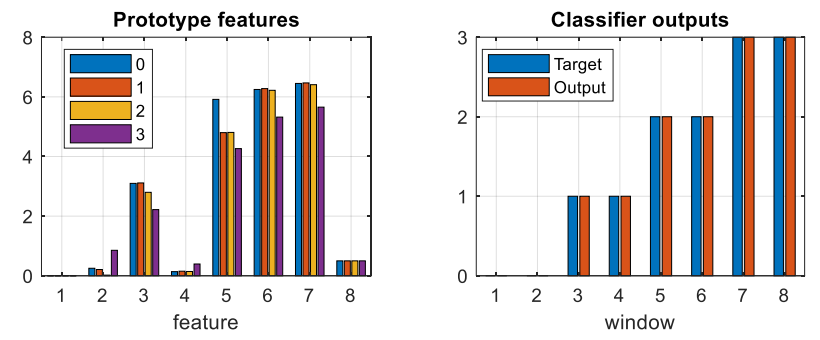

Fig. 7. Results of the diagnosis by using synthetic signals in testing

For the case of the real test signals, the parameters $N s$ and $N w$ are dramatically changed to obtain right results. Fig. 8 shows the results of the diagnosis for fixed window, $N w=$ 2,500, and Fig. 9 the results for fixed model size, $N s=4,000$. In all cases, the Manhattan distance was used for classification.

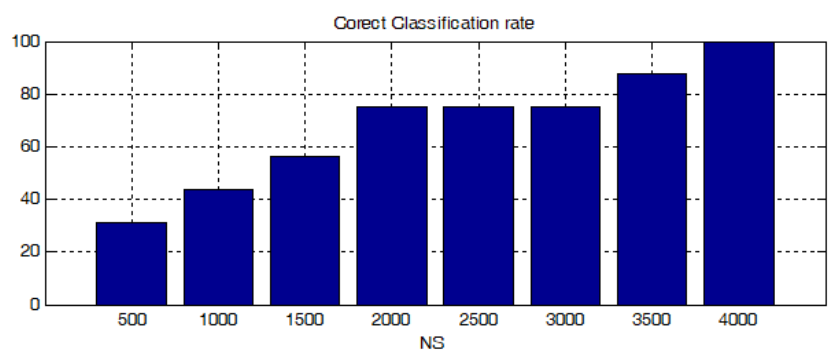

Fig. 8. Results of the diagnosis by real signals; fixed $N w=2500$

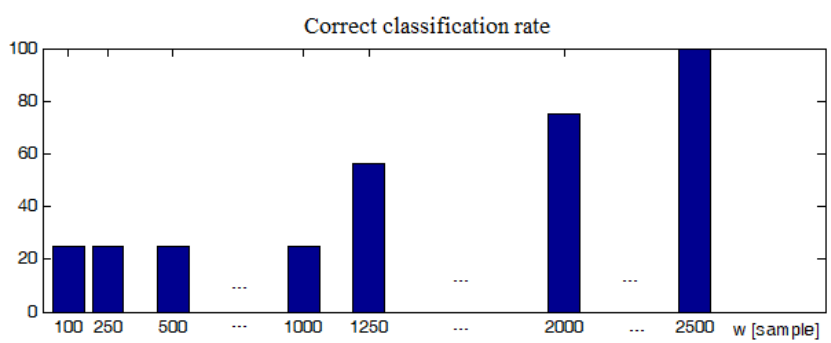

Fig. 9. Results of the diagnosis by using real signals; fixed $N S=4,000$

Fig. 10 presents the results of the correlation-based technique, used as an alternative or reference for the above methods. Three algorithms were tested based on: (1) correlation of the probability distributions; (2) correlation of samples of the signals; (3) Euclidean distance between distributions of the probabilities. The best results are obtained with correlation of the signals, for which a window of length 2,000 samples is enough to obtain the perfect classification. For the synthetic signals, a window length of 500 samples generates a rate of $100 \%$ for each of the three algorithms.

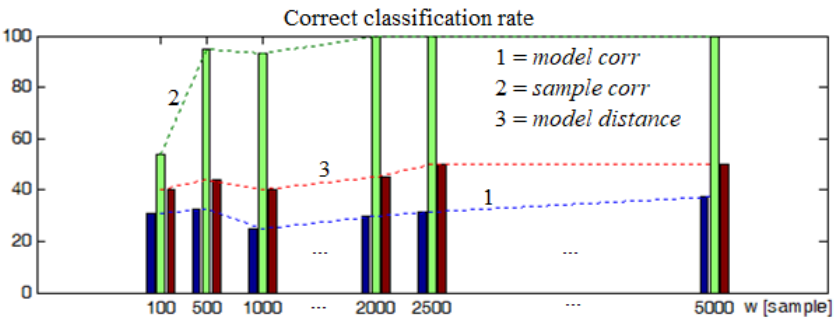

Fig. 10. Results of the diagnosis by using real signals; correlation based 
The last approach, based on Renyi divergence, provides good results $(100 \%$ RR) only for synthetic signals and about $50 \%$ recognition rate regardless of the size of the model or the observation window.

\section{CONCLUSION}

The objective of this work was to explore the detection and classification of faults in bearings, by using information-based features, in terms of computation, selection and classification.

Data coming from vibration signals are considered. Raw data are explored in frames/windows, with a variable length imposed by the accuracy of the change detection algorithms, which requires high lengths for the efficient estimations of the parameters and - finally - for the performance of the change detection and diagnosis. The basic set of features is the source model of the analyzed signals, i.e., the probabilities of the symbols. Next, average values for entropy and complexity measures are included, up to five variables. The complete set of features uses three statistical moments, i.e., variance, skewness and kurtosis applied to the set of the probabilities.

The simulation results with real physical data reveal a solution based on high values for the source model $(4,000$ elements) and for wide windows (of length 2,500). As alternative to this approach, the correlation-based method uses a window of length 2,000 samples.

The method based on information modeling is still superior, by generality and by revealing supplementary aspects, e.g., data complexity.

An interesting study is to extend the tested set of features with the information-based features from frequency domain.

\section{REFERENCES}

[1] D. Aiordachioaie, "Industrial Process Diagnosis based on Information and Time Domain Models," 2021 13th International Conference on Electronics, Computers and Artificial Intelligence (ECAI)," Pitesti, Romania, pp. 1-4.

[2] J. Chen, C. Lin, D. Peng and H. Ge, "Fault Diagnosis of Rotating Machinery: A Review and Bibliometric Analysis," in IEEE Access, vol. 8, pp. 224985-225003, 2020.

[3] R. Isermann, Fault-Diagnosis Applications Model-Based Condition Monitoring: Actuators, Drives, Machinery, Plants, Sensors, and Faulttolerant Systems, Springer, 2011.

[4] J. Gertler, Fault Detection and Diagnosis in Engineering Systems, Marcel Dekker, 1998.

[5] J. Chen and R.J. Patton, Robust Model-Based Fault Diagnosis for Dynamic Systems, Kluwer Academic Publishers, 1999.

[6] L. H. Chiang, E.L. Russell, and R. D. Braatz, Fault detection and diagnosis in industrial systems, Springer-Verlag London 2001.

[7] A. Abid, M.T. Khan, and J. Iqbal, "A review on fault detection and diagnosis techniques: basics and beyond," Artif Intell Rev 54, 36393664.

[8] Y. Zhang, L. Zhang, and H. Zhang, "Fault Detection for Industrial Processes", Mathematical Problems in Engineering, Hindawi Publishing Corporation, Vol. 2012, Article ID 757828, 18 pages.

[9] G. Yu, T. Lin, Z. Wang and Y. Li, "Time-Reassigned Multisynchrosqueezing Transform for Bearing Fault Diagnosis of Rotating Machinery," in IEEE Trans. on Ind. Electr., vol. 68, no. 2, pp. 1486-1496.

[10] X. Tu, Q. Zhang, Z. He, Y. Hu, S. Abbas and F. Li, "Generalized Horizontal Synchrosqueezing Transform: Algorithm and Applications," in IEEE Trans. on Ind. Electr., vol. 68, no. 6, June 2021, pp. 5293-5302.

[11] Th.D. Popescu and D. Aiordăchioaie, New Procedure for Change Detection Operating on Rényi Entropy with Application in Seismic Signals Processing, Circuits Syst Signal Process, 36, 2017, 3778-3798.
[12] D. Aiordachioaie, "On change detection and process diagnosis based on information and frequency domain models", presented at International Scientific Conference on Communications, Information, Electronic and Energy Systems (CIEES), Varna, Bulgaria, 2021.

[13] D. Aiordachioaie and Th. D. Popescu, "Change Detection by Feature Extraction and Processing from Time-Frequency Images," 2018 10th Int. Conf. on Electronics, Computers and Artificial Intelligence (ECAI), 2018, pp. 1-7.

[14] D. Aiordachioaie and Th. D. Popescu, "Aspects of Time Series Analysis with Entropies and Complexity Measures," 2020 Int. Symp. on Electronics and Telecommunications (ISETC), 2020, pp. 1-4.

[15] D. Aiordachioaie, Th. D. Popescu and S. Marius Pavel, "On Change Detection in the Complexity of the Time Series with Multiscale Renyi Entropy Processing," 2020 24th Int. Conf. on System Theory, Control and Computing (ICSTCC), 2020, pp. 927-932.

[16] Ji, C., Ma, F., Wang, J., Wang, J., Sun,W., "Real-Time Industrial Process Fault Diagnosis Based on Time Delayed Mutual Information Analysis", Processes, 2021, 9, 1027.

[17] S. Zhou, J. Zhang, and S. Wang, "Fault Diagnosis in Industrial Processes Using Principal Component Analysis and Hidden Markov Model", Proc. of the 2004 American Control Conference, Boston, Massachusetts, June 30 - July 2, pp. 5680-5685.

[18] J. Zhu, M. Jiang, Z. Liu, "Fault Detection and Diagnosis in Industrial Processes with Variational Autoencoder: A Comprehensive Study", Sensors, 2022, 22, 227.

[19] S. Verron, T. Tiplica, and A. Kobi, "Fault diagnosis of industrial systems by conditional Gaussian network including a distance rejection criterion", Eng. Appl. of Artificial Intelligence, Elsevier, 2010, 23 (7), pp.1229-1235.

[20] R. Liu, B. Yang, E. Zio, and Xuefeng Chen, "Artificial intelligence for fault diagnosis of rotating machinery: A review," Mechanical Systems and Signal Processing, 108, 2018, pp. 33-47.

[21] Y. Li, S. Wang, N. Li and Z. Deng, "Multiscale Symbolic Diversity Entropy: A Novel Measurement Approach for Time-Series Analysis and Its Application in Fault Diagnosis of Planetary Gearboxes," in IEEE Trans. on Industrial Informatics, vol. 18, no. 2, pp. 1121-1131.

[22] Q. Lu, B. Jiang and E. Harinath, "Fault Diagnosis in Industrial Processes by Maximizing Pairwise Kullback-Leibler Divergence," in IEEE Trans. on Control Systems Technology, vol. 29, no. 2, pp. 780785.

[23] Q. Wang, Y. Xiao, S. Wang, W. Liu and X. Liu, "A Method for Constructing Automatic Rolling Bearing Fault Identification Model Based on Refined Composite Multi-Scale Dispersion Entropy," in IEEE Access, vol. 9, 2021, pp. 86412-86428.

[24] J. Wang, D. Wang, S. Wang, W. Li and K. Song, "Fault Diagnosis of Bearings Based on Multi-Sensor Information Fusion and 2D Convolutional Neural Network," in IEEE Access, vol. 9, 2021, pp. 23717-23725.

[25] D. Scott, C. Shang, B. Huang and D. Huang, "A Holistic Probabilistic Framework for Monitoring Nonstationary Dynamic Industrial Processes," in IEEE Trans. on Control Systems Technology, vol. 29, no. 5, 2021, pp. 2239-2246.

[26] X. Pu and C. Li, "Probabilistic Information-Theoretic Discriminant Analysis for Industrial Label-Noise Fault Diagnosis," in IEEE Trans. on Ind. Informatics, vol. 17, no. 4, 2021, pp. 2664-2674.

[27] I.El-Thalji and E. Jantunen, "A summary of fault modelling and predictive health monitoring of rolling element bearings," Mechanical Systems and Signal Processing, 60-61, 2015, pp. 252-272.

[28] R. B. Randal, Vibration-based condition monitoring: industrial, aerospace and automotive applications, John Wiley \& Sons, 2011.

[29] R.B. Randall and J. Antoni, "Rolling element bearing diagnostics-A tutorial," Mechanical Syst. and Signal Proc., 25, 2011, pp. 485-520.

[30] Th. D. Popescu, D. Aiordachioaie and M. Manolescu, "Change detection in vibration analysis - A review of problems and solutions," 2017 5th Int. Symp. on Electrical and Electronics Engineering (ISEEE), Galati, Romania, 2017, pp. 1-6.

[31] A. Rai and S.H. Upadhyay, "A review on signal processing techniques utilized in the fault diagnosis of rolling element bearings," Tribology International, 96, 2016, pp. 289-306.

[32] M. Cerrada, R.V. Sánchez, C. Li, F. Pacheco, D. Cabrera, J.V. de Oliveira, and R.E.Vásquez, "A review on data-driven fault severity assessment in rolling bearings," Mechanical Systems and Signal Processing, 99, 2018, pp. 169-196. 
[33] L. He, L. Hao and W. Qiao, "Remote Monitoring and Diagnostics of Pitch-Bearing Defects in an MW-Scale Wind Turbine Using Pitch Symmetrical-Component Analysis," in IEEE Trans. on Ind. Appl., vol. 57, no. 4, 2021, pp. 3252-3261.

[34] T. Wang, Z. Liu, G. Lu and J. Liu, "Temporal-Spatio Graph Based Spectrum Analysis for Bearing Fault Detection and Diagnosis," in IEEE Trans. on Ind. Electr., vol. 68, no. 3, 2021, pp. 2598-2607.

[35] G. Zhang, Y. Shu and T. Zhang, "Adaptive Modulation Stochastic Resonance of Two-Dimensional Asymmetric Bistable System and its Application in Bearing Fault Diagnosis," in IEEE Access, vol. 9, 2021, pp. 104722-104733.

[36] M. T. Pham, J. -M. Kim and C. H. Kim, "Efficient Fault Diagnosis of Rolling Bearings Using Neural Network Architecture Search and Sharing Weights," in IEEE Access, vol. 9, 2021, pp. 98800-98811.

[37] Y. Lu, Z. Wang, D. Zhu, Q. Gao and D. Sun, "Bearing Fault Diagnosis Based on Clustering and Sparse Representation in Frequency Domain," in IEEE Trans. on Instr. and Measurement, vol. 70, 2021, pp. 1-14.

[38] C.Sobie, C.Freitas, and M. Nicolai, "Simulation-driven machine learning: Bearing fault classification," Mechanical Systems and Signal Processing, vol. 99, 2018, pp. 403-419.

[39] M. Gan, C. Wang, and C. Zhu, "Construction of hierarchical diagnosis network based on deep learning and its application in the fault pattern recognition of rolling element bearings," Mechanical Systems and Signal Processing, vol. 72-73, 2016, pp. 92-104.

[40] H. Shao, H. Jiang, F. Wang, Y. Wang, "Rolling bearing fault diagnosis using adaptive deep belief network with dual-tree complex wavelet packet,“ ISA Transactions, vol. 69, 2017, pp. 187-201.

[41] Z. Feng, M. Liang, and F. Chu, "Recent advances in time-frequency analysis methods for machinery fault diagnosis: A review with application examples," Mechanical Systems and Signal Processing, vol. 38, 2013, pp. 165-2015.

[42] C. Li and M. Liang, "Time-frequency signal analysis for gearbox fault diagnosis using a generalized synchrosqueezing transform,"
Mechanical Systems and Signal Processing, vol. 26, 2012, pp. 205217.

[43] M. Aboy, R. Hornero, D. Abásolo, and D. Alvarez, "Interpretation of the Lempel-Ziv complexity measure in the context of biomedical signal analysis," IEEE Trans. on Bio-med. Eng., vol. 53, 2006, pp. 2282-2288.

[44] J.R. Pierce, An introduction to information theory: symbols, signals and noise, Dover 1980.

[45] A. Rényi, "On Measures of Entropy and Information," Proc. the $4^{\text {th }}$ Berkeley Symp. on Math Statistics and Prob., Vol1: Contributions to the Theory of Statistics, 547-561, Univ. of California, Berkeley, 1961.

[46] M. Costa, A.L. Goldberger, and C.K. Peng, "Multiscale entropy analysis of complex physiologic time series," Phys. Rev. Lett., 89, 068102, 2002

[47] A. Humeau-Heurtier, "The Multiscale Entropy Algorithm and Its Variants: A review," Entropy, vol 17, 2015, pp. 3110-3123.

[48] A. Anastasiadis, "Special Issue: Tsallis Entropy," Entropy, 14, no. 2, 2012, pp. 174-176.

[49] T. Maszczyk and W. Duch, "Comparison of Shannon, Renyi and Tsallis entropy used in decision trees," Lecture Notes in Computer Science, vol. 5097, 2008, pp. 643-651.

[50] H. Neemuchwala, A. Hero, and P. Carson, "Image matching using alpha-entropy meas. and entropic graphs," Signal Processing, 85, 2005, pp. 277-296.

[51] M.H. Hayes, Statistical Digital Signal Processing and Modeling, John Wiley \& Sons, 1996.

[52] T. van Erven and P. Harremos, "Rényi Divergence and KullbackLeibler Divergence," in IEEE Trans. on Information Theory, vol. 60, no. 7, 2014, pp. 3797-3820.

[53] Case Western Reserve University Bearing Data Center, Available at: http://csegroups.case.edu/bearingdatacenter/home, 2019.

[54] W.A. Smith and R.B. Randall, "Rolling element bearing diagnostics using the Case Western Reserve University data: A benchmark study," Mechanical Systems and Signal Processing, 64-65, 2015, pp. 100.-131. 